Spring 2018

\title{
The Use of Prasugrel and Ticagrelor in Pipeline Flow Diversion
}

\author{
Elias Atallah, MD \\ Thomas Jefferson University \\ Hassan Saad, MD \\ Thomas Jefferson University \\ Kimon Bekelis, MD \\ Thomas Jefferson University \\ Nohra Chalouhi, MD \\ Thomas Jefferson University \\ Stavropoula Tjoumakaris, MD \\ Thomas Jefferson University
}

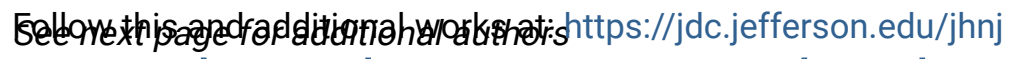 Let us know how access to this document benefits you}

\author{
Recommended Citation \\ Atallah, MD, Elias; Saad, MD, Hassan; Bekelis, MD, Kimon; Chalouhi, MD, Nohra; Tjoumakaris, MD, \\ Stavropoula; Hasan, MD, David; Eller, MD, Gorge; Stidd, MD, David; Rosenwasser, MD, MBA, FACS, FAHA \\ MD, Robert H.; and Jabbour, Pascal MD (2018) "The Use of Prasugrel and Ticagrelor in Pipeline Flow \\ Diversion," JHN Journal: Vol. 13 : Iss. 2 , Article 5. \\ DOI: https://doi.org/10.29046/JHNJ.013.2.005 \\ Available at: https://jdc.jefferson.edu/jhnj/vol13/iss2/5
}

This Article is brought to you for free and open access by the Jefferson Digital Commons. The Jefferson Digital Commons is a service of Thomas Jefferson University's Center for Teaching and Learning (CTL). The Commons is a showcase for Jefferson books and journals, peer-reviewed scholarly publications, unique historical collections from the University archives, and teaching tools. The Jefferson Digital Commons allows researchers and interested readers anywhere in the world to learn about and keep up to date with Jefferson scholarship. This article has been accepted for inclusion in JHN Journal by an authorized administrator of the Jefferson Digital Commons. For more information, please contact: JeffersonDigitalCommons@jefferson.edu. 


\section{The Use of Prasugrel and Ticagrelor in Pipeline Flow Diversion}

\section{Authors}

Elias Atallah, MD; Hassan Saad, MD; Kimon Bekelis, MD; Nohra Chalouhi, MD; Stavropoula Tjoumakaris, MD; David Hasan, MD; Gorge Eller, MD; David Stidd, MD; Robert H. Rosenwasser, MD, MBA, FACS, FAHA MD; and Pascal Jabbour MD 


\section{The Use of Prasugrel and Ticagrelor in Pipeline Flow Diversion}

Elias Atallah, MD; Hassan Saad, MD; Kimon Bekelis, MD; Nohra Chalouhi, MD; Stavropoula Tjoumakaris, MD; David Hasan, MD; Gorge Eller, MD; David Stidd MD, Robert H. Rosenwasser, MD, MBA, FACS, FAHA; Pascal Jabbour, MD

Department of Neurological Surgery, Thomas Jefferson University, Philadelphia, PA

\begin{abstract}
Background: Despite the routine clopidogrel/aspirin anti-platelet therapy, complications like thromboembolism, continue to be encountered with PED. We studied the safety and the efficacy of prasugrel in the management of clopidogrel non-responders treated for intracranial aneurysms.
\end{abstract}

Methods: 437 consecutive neurosurgery patients were identified between January 2011 and May 2016. Patients allergic or having $<30 \%$ platelet-inhibition with a daily $75 \mathrm{mg}$ of clopidogrel were dispensed $10 \mathrm{mg}$ of prasugrel daily $(n=20)$ or $90 \mathrm{mg}$ of ticagrelor twice daily $(n=2)$. The average follow-up was 15.8 months (SD=12.4 months). Patient clinical well being was evaluated with the modified Rankin Scale (mRS) registered before the discharge and at each follow-up visit. To control confounding we used multivariable mixed-effects logistic regression and propensity score conditioning.

Results: 26 of $437(5.9 \%$ ) patients (mean of age 56.3 years; 62 women [14,2\%]) presented with a sub-arachnoid hemorrhage. 1 patient was allergic to clopidogrel and prasugrel simultaneously. All the patients receiving prasugrel $(n=22)$ had a $m R S<2$ on their latest follow-up visit (mean=0.67; SD=1.15). In a multivariate analysis, clopidogrel did not affect the $\mathrm{mRS}$ on last follow-up, $\mathrm{p}=0.14$. Multivariable logistic regression showed that clopidogrel was not associated with an increased long-term recurrence rate (odds ratio[OR], 0.17; 95\%Confidence Interval [CI95\%], 0.01-2.70; $p=0.21$ ) neither with an increased thromboembolic accident rate (OR, 0.46; C195\%, 0.12-1.67; $p=0.36$ ) nor with an increased hemorrhagic event rate (OR, 0.39; CI95\%,0.91-1.64; $\mathrm{p}=0.20)$. None of the patients receiving prasugrel deceased or had a long-term recurrence nor a hemorrhagic event, only 1 patient suffered from mild aphasia subsequent to a thromboembolic event. 3 patients on clopidogrel passed during the study: (2) from acute SAH and (1) from intra-parenchymal hemorrhage. Clopidogrel was not associated with an increased mortality rate (OR, 2.18; C195\%,0.11-43.27; $\mathrm{p}=0.61)$. The same associations were present in propensity score adjusted models.

Conclusion: In a cohort of patients treated with PED for their intracranial aneurysms, prasugrel $(10 \mathrm{mg} /$ day) is a safe alternative to clopidogrel resistant, allergic or non-responders.

\section{INTRODUCTION}

Since the 2011 FDA approval, PED has been a favored option in treating cerebral aneurysm(s). ${ }^{15}$ The PED is a self-expanding stent with $30-35 \%$ metal surface area coverage that diverts blood flow from the aneurysm lumen to the downstream arteries causing aneurysm sac thrombosis. ${ }^{3}$ However, there is a window period until full luminal endothelialization of the PED occurs, during which the patient is at a high risk of thromboembolic events. ${ }^{112,13}$ The use of Dual Anti-Platelet Therapy (DAPT) with aspirin and clopidogrel has been recommended for preventing thrombotic and hemorrhagic complications that occur after the deployment of PEDs. ${ }^{12}$ However, Delgado Almandoz JE et al. demonstrated that thromboembolic complications continue to be encountered, particularly with PED, despite the routine DAPT. Approximately 30\% of patients exhibit anti-platelet resistance. ${ }^{1}$ Insufficient platelet inhibition in CYP2C19 heterozygotes causes this variability in the response to clopidogrel. Several centers have replaced clopidogrel with different anti-aggregation drugs like prasugrel or ticagrelor in the management of these resistant cases. 5,14 Prasugrel and Ticagrelor achieve more potent and rapid inhibition of platelet aggregation and decreased intersubject response variability. ${ }^{6,8}$ In our Study, we identified all the patients that were resistant to clopidogrel. They were dispensed prasugrel or ticagrelor in order to achieve the optimal platelet inhibition. This allowed them to undertake their flow diverting stent treatment. We demonstrate the safety and efficacy of prasugrel and ticagrelor as alternative antiplatelet agents whilst dispensed in conjunction with aspirin in clopidogrel non-responders. 
Table 1. Association between outcomes and clopidogrel

\begin{tabular}{|c|c|c|c|c|c|c|c|c|c|c|}
\hline \multirow[t]{2}{*}{ Model } & \multicolumn{2}{|c|}{$\begin{array}{l}\text { Long-term } \\
\text { recurrence }\end{array}$} & \multicolumn{2}{|c|}{ Intra-PED stenosis } & \multicolumn{2}{|c|}{$\begin{array}{c}\text { Thromboembolic } \\
\text { Complications }\end{array}$} & \multicolumn{2}{|c|}{$\begin{array}{l}\text { Hemorrhagic } \\
\text { Complications }\end{array}$} & \multicolumn{2}{|c|}{ Mortality } \\
\hline & OR(Cl95\%) & $p$ value & OR(Cl95\%) & $p$ value & OR(CI95\%) & $p$ value & OR(Cl95\%) & $p$ value & OR(Cl95\%) & $p$ value \\
\hline $\begin{array}{l}\text { Multi-variable } \\
\text { regression }\end{array}$ & $\begin{array}{l}0.17(0.01 \\
\text { to } 2.70)\end{array}$ & 0.21 & $\begin{array}{l}0.44(0.09 \text { to } \\
2.15)\end{array}$ & 0.31 & $\begin{array}{c}0.46(0.12 \\
\text { to } 1.67)\end{array}$ & 0.24 & $\begin{array}{l}0.39(0.91 \\
\text { to } 1.64)\end{array}$ & 0.20 & $\begin{array}{l}2.18(0.11 \\
\text { to } 43.27)\end{array}$ & 0.61 \\
\hline $\begin{array}{l}\text { Propensity score } \\
\text { adjustment }\end{array}$ & $\begin{array}{l}0.26(0.22 \\
\text { to } 3.03)\end{array}$ & 0.28 & $\begin{array}{c}0.46(0.09 \text { to } \\
2.44)\end{array}$ & 0.37 & $\begin{array}{c}0.39(0.11 \\
\text { to } 1.41)\end{array}$ & 0.82 & $\begin{array}{c}0.33(0,08 \\
\text { to } 1.37)\end{array}$ & 0.13 & $\begin{array}{c}0.73(0.75 \\
\text { to } 7.17)\end{array}$ & 0.79 \\
\hline
\end{tabular}

\section{METHODS}

\section{Cohort creation}

We performed a retrospective cohort study of all patients undergoing treatment of cerebral aneurysms with flow diverting stents in a tertiary referral center between January 2011 and May 2016. The Institutional Review Board reviewed and approved the study protocol. All patients received a pipeline embolization device (PED; Covidien, Irvine, California). The characteristics of the cohort at the baseline can be seen in Table 1 .

\section{Treatment protocol}

In our institution, patients are pretreated with $75 \mathrm{mg}$ of clopidogrel daily and at least $81 \mathrm{mg}$ of aspirin daily for 10 days before their PED deployment. Some patients presenting for their intervention without having received the appropriate 10 days DAPT were loaded with 325-650mg of aspirin and a bolus of anti-aggregate drug (600mg of clopidogrel or 40-60mg of prasugrel or $90 \mathrm{mg}$ of ticagrelor) within less than 24 hours to their intervention. We routinely calculated the P2Y12 platelet inhibition assay (VerifyNow; Accumetrics, San Diego, California) for all the patients before the procedure. Prasugrel was considered when patients are allergic, non-responders or resistant to clopidogrel. Resistance was defined as having $(<30 \%)$ of platelets P2Y12 receptor inhibition. 22 patients among the 437 did not have a significant platelet inhibition (<30\%) with clopidogrel, they constituted our population. Ticagrelor was the final alternative for those whose P2Y12 platelet inhibition was still not satisfactory with prasugrel. Patients were continued after the operation on $75 \mathrm{mg}$ of clopidogrel daily or $10 \mathrm{mg}$ of prasugrel daily or $90 \mathrm{mg}$ of Ticagrelor twice daily. Prophylactic anti-platelets therapy was given as a minimum of 6 months to 1 year following the procedure.

\section{Outcome variables}

The key primary outcome was the patients' Modified Rankin Scale which was calculated and registered before the discharge of the patients and at each follow-up visit. Mortality, thromboembolic events and DSA documented hemorrhagic accidents, following the aneurysm's pipeline treatment, were considered primary outcomes. Secondary outcomes were the post interventional length of stay and the long-term recurrence.

\section{Patient Follow Up}

Patients were scheduled for a follow up visit with the senior author after 1, 3, 6 and 18 months following their discharge from the institution.

The efficacy and the safety of the prophylactic DAPT post pipeline treatment were evaluated on initial postoperative angiography and follow-up angiography when available. Cerebrovascular Angiography (digital subtraction angiography DSA) was required at their 6 months visit and then patients were followed accordingly to evaluate for bleeding recurrence or vessel stenosis. Additional information on the number of PED used for initial treatment and on the stent migration were all collected during the follow up (Table 1). Head computed tomography scans were compared and analyzed by the senior author to document any new or recurrent subarachnoid or intra-parenchymal hemorrhage, only if the patients were to develop new insidious symptoms. Medical charts were reviewed to determine whether any retroperitoneal, gastrointestinal, or genitourinary bleeding had occurred.

\section{Exposure variables}

The primary exposure variable was the treatment (prasugrel or ticagrelor vs clopidogrel).

Covariates used for risk adjustment were age and gender. The comorbidities used for risk adjustment were: hypertension, diabetes mellitus, smoking, previous sub arachnoid hemorrhage, aneurysm size, per-procedural balloon, previous coiling.

\section{Statistical analysis}

To investigate the association of clopidogrel exposure and our outcome measures we used several methods to address confounding. Initially, for binary outcomes we used a multivariable logistic regression controlling for all the covariates mentioned above. In order to control for clustering at the physician level, we employed mixed effect models with physician as a random effects variable. For continuous outcomes, we used the corresponding linear regression analyses.

In an alternate way to control for confounding for binary outcomes we employed a propensity score adjusted logistic regression model. To derive the propensity of receiving clopidogrel we developed a prediction model using logistic regression, based on all the covariates described above. We subsequently employed a logistic regression with adjustment (stratification) by quantiles (we chose the number of quantiles to be 10) of the propensity score. Operating physician was again used as a random effects variable. 


\section{Table 2. Patient characteristics}

\begin{tabular}{|l|c|c|c|c|}
\hline \multirow{2}{*}{} & \multicolumn{2}{|c|}{ Clopidogrel } & \multicolumn{2}{c|}{ Prasugrel - Ticagrelor } \\
\cline { 2 - 5 } & \multicolumn{2}{|c|}{374} & \multicolumn{2}{c|}{22} \\
\hline No & Mean & SD & Mean & SD \\
\hline Age (years) & 56.25 & 13.29 & 57.31 & 13.55 \\
\hline Sex & 1.15 & 0.36 & 1.23 & 0.43 \\
\hline Hypertension & 0.51 & 0.50 & 0.54 & 0.51 \\
\hline Smoking & 0.50 & 0.50 & 0.65 & 0.49 \\
\hline Aneurysm size (mm) & 9.11 & 5.87 & 12.93 & 10.93 \\
\hline Previous SAH & 0.10 & 0.30 & 0.15 & 0.37 \\
\hline Adjunctive Coiling & 0.74 & 0.26 & 0.08 & 0.27 \\
\hline Stent migration & 0.01 & 0.09 & 0.04 & 0.19 \\
\hline $\begin{array}{l}\text { Number of stents per } \\
\text { patient }\end{array}$ & 1.21 & 0.56 & 1.32 & 0.57 \\
\hline mRS on last follow up & 0.32 & 0.75 & 0.67 & 1.15 \\
\hline
\end{tabular}

Patients who were lost to follow up were not included in the original analysis. In sensitivity analysis, all the above analyses were repeated used multiple imputations for the patients lost to follow up. We created 5 imputed datasets. The directions of the observed associations did not change and these results are not reported further.

Regression diagnostics were performed for all analyses. Given that the long-term recurrence was $2 \%$ in a study sample of 437 patients, we had an $80 \%$ power to detect a difference in long-term recurrence as small as $13.4 \%$, at an $\alpha$-level of 0.05 . All probability values were the result of two sided tests. Stata version 13 (StataCorp, College Station, TX) was used for statistical analysis.

\section{RESULTS}

\section{Demographic characteristics}

Between 2011 and 2016, a total of 437 patients (mean of age 56.3 years; 62 women $[14,2 \%]$ ) underwent treatment with PED in our institution. 26 (5.9\%) patients presented with an acute subarachnoid hemorrhage (SAH). 374 received clopidogrel [361 with aspirin, 9 with Coumadin, 4 with rivaroxaban], $20(4.6 \% ;$ Mean $=0.047 ; \mathrm{SD}=0.2117)$ received prasugrel and 2 received ticagrelor (Mean $=0.0074 ; \mathrm{SD}=0.0858$ ). 7 patients were lost for follow-up after their intervention ( 6 from the clopidogrel group and 1 from the prasugrel group). 1 patient was reported allergic to clopidogrel and prasugrel. (Table 1)

\section{Efficacy of prasugrel and ticagrelor}

a. Intra-pipeline stenosis

Of 369 patients receiving clopidogrel, 23(6.1\%) of the patients had an intra-PED stenosis.

In the group of patients receiving prasugrel \& ticagrelor the mean of intra-pipeline stenosis was $0.117(S D=0.33)$, only 2 patients had an intra-pipeline stenosis: $1(5 \%)$ receiving prasugrel the other ticagrelor. The mean of intra-pipeline stenosis with patients receiving clopidogrel was 0.071(6.7\%; $S D=0.26)$. A univariate analysis of the effect of clopidogrel on the thromboembolic complication rate did not show any correlation between the two variables (OR, 0.58 ; $C 195 \%, 0.12$ to $2.70 ; p=0.48$ ). In a multivariable mixed-effects logistic regression, clopidogrel was not associated with an increased intra-pipeline stenosis (OR, 0.44; Cl95\%,0.08 to 2.15; $p=0.31$ ). This was coherent with the propensity score adjusted model. (OR, 0.46; $\mathrm{Cl} 195 \%, 0.088$ to $2.44 ; \mathrm{p}=0.37$ ).

\section{b. Long term recurrence}

None of the 22 patients receiving prasugrel or ticagrelor had a long term recurrence. Of 374 patients receiving clopidogrel, $1,6 \%$ suffered from a long term recurrence rate with a mean of $0.02(S D=0.13)$. A univariate analysis of the effect of clopidogrel on the long term recurrence rate does not show any correlation between the two variables (OR, 0.27; C195\%, 0.03 to $2.41 ; p=0.24)$. In a multivariable mixed-effects logistic regression, clopidogrel was not associated with an increased long term recurrence (OR, 0.17; Cl95\%, 0.01 to 2.70; $p=0.21$ ). This was consistent with the propensity score adjusted model (OR, 0.26; C195\%, 0.02 to 3.03; $p=0.28$ ). (Table.2)

\section{Safety of prasugrel and ticagrelor}

\section{c. Post PED complications}

Of 22 patients receiving prasugrel and ticagrelor the mean of postprocedural complications is 0.19 $(\mathrm{SD}=0.40)$, only 1 patient developed an arterio-venous V3 fistula and 1 other patient had an ophtalmoplegia and a ptosis of the left eye. Of 374 patients receiving Clopidogrel, the mean post PED complications was $0,53(S D=0.23)$. A univariate analysis of the effect of clopidogrel on the post pipeline complication rate is associated with an increased post pipeline complication rate (OR, $0.24 ; \mathrm{Cl} 195 \%, 0.08$ to $0.70 ; \mathrm{p}=0.01$ ). In a multivariable mixed-effects logistic regression, clopidogrel is also associated with an increased post pipeline complication rate (OR, 0.28; Cl95\%, 0.08 to 1.01; $\mathrm{p}=0.05$ ). This was consistent with the propensity score adjusted model where $p$-value was slightly superior to 0.05 (OR, 0.27; C195\%, 0.07 to 1.03; $p=0.055)$. 


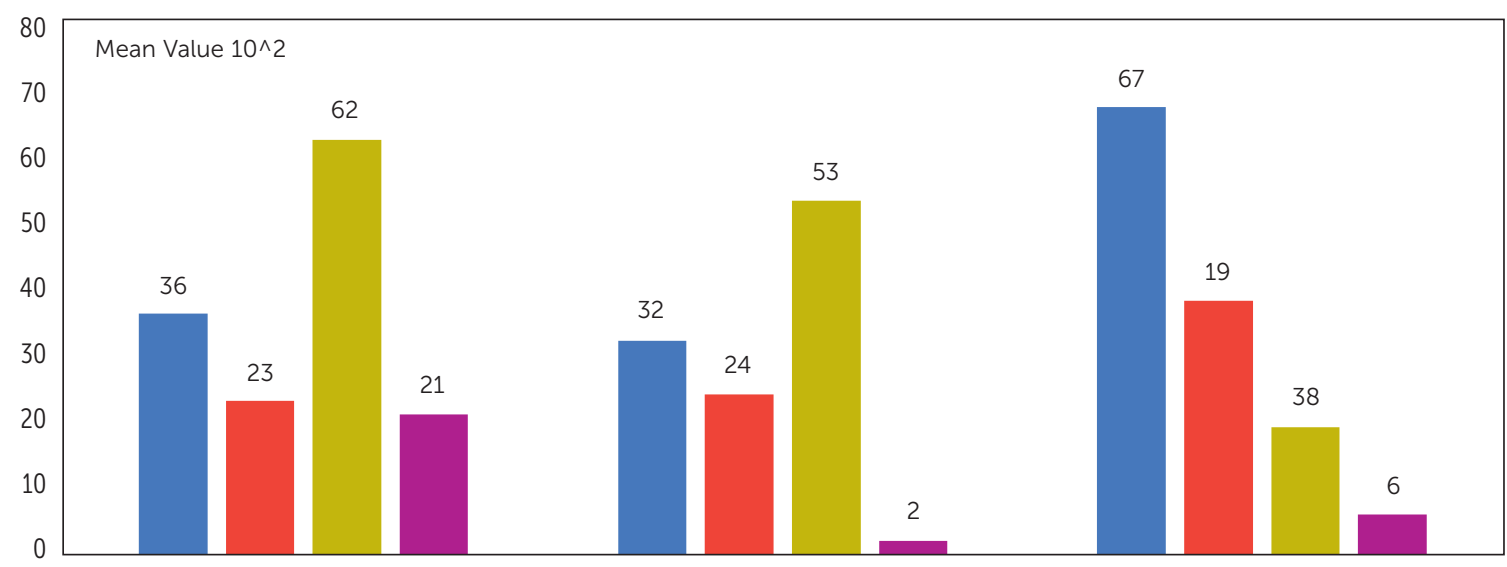

$\square$ Latest MRS $\square$ Mortality $\square$ Post PED Complications $\square$ Long Term Recurrence

Figure 1. Graph showing the mean values of the clinical outcomes according to the prescribed antiplatelet drug

\section{d. Thromboembolic complications}

Of 374 patients prescribed clopidogrel, the mean of thromboembolic events was $0.72(\mathrm{SD}=0.26)$. While 28 (7.4\%) patients receiving clopidogrel had thromboembolic complications, only 1 patient dispensed prasugrel suffered from word finding difficulty. A univariate analysis of the effect of clopidogrel on the thromboembolic complication rate does not show any correlation between the two variables (OR, 0.43; CI95\%, 0.14 to 1.32; $\mathrm{p}=0.14)$. We found similar results in a multivariable mixed-effects logistic regression (OR, 0.46; CI95\%, 0.12 to 1.67; $p=0.36$ ) and a propensity score adjusted model (OR, 0.39; Cl95\%, 0.11 to $1.41 ; p=0.82$ ).

\section{e. Hemorrhagic complications}

None of the patients receiving prasugrel or ticagrelor suffered from hemorrhagic complication. Of 374 patients receiving clopidogrel the mean of the hemorrhagic complications was $0.45(5.6 \% ; \mathrm{SD}=0.21)$. A univariate analysis of the effect of clopidogrel on the hemorrhagic complication rate does not show any correlation between the two variables (OR, 0.36; Cl95\%, 0.10 to $1.33 ; p=0.13)$. In a multivariable mixed effect logistic regression, clopidogrel was not associated with an increased hemorrhagic event rate (OR, 0.39; C195\%, 0.91 to 1.64; $p=0.20$ ). We found similar results with the propensity score adjusted model (OR, 0.33; Cl95\%, 0.08 to 1.37; $p=0.13$ )

\section{f. Mortality}

Patients receiving clopidogrel had a mean mortality rate $0.02(2.67 \%$; $\mathrm{SD}=0.15$ ). (Figure.1) 9 patients were lost: 6 patients dying from various non PED related events such as severe sepsis (1), malignant hypertension with large middle cerebral artery infarct (1), severe gastro-intestinal complication (1), non reported cause of death (3). Only 3 patients from this group were announced dead from acute SAH (2) and intra-parenchymal hemorrhage (1). None of the patients receiving prasugrel and ticagrelor were lost. A univariate analysis of the effect of clopidogrel on the mortality rate does not show any correlation between the two variables, (OR, 0.61; C195\%, 0.75 to $5.03 ; p=0.65$ ). In a multivariable mixed effect logistic regression, clopidogrel was not associated with an increased mortality rate (OR, 2.18; CI95\%, 0.11 to $43.27 ; p=0.61$ ). This persisted in a propensity score adjusted model (OR, 0.73; Cl95\%,0.75 to $7.17 ; \mathrm{p}=0.79$ ). (Table.2)

\section{g. Latest clinical status}

Of 22 patients receiving prasugrel or ticagrelor, All the patients had a $\mathrm{mRS}<=2$ on their latest follow-up visit with a mean of $0.67(S D=1.15)$. $98.4 \%$ of 374 patients receiving clopidogrel had a $\mathrm{mRS}=<2$ on their latest follow-up visit with a mean of $0.32(\mathrm{SD}=0.75)$. (Figure.1) in a multivariate analysis were the latest $\mathrm{mRS}$ is a dependent variable, clopidogrel did not affect the mRS score on last follow-up, $p=0.14$. (Figure.1)

\section{h. Post interventional hospital stay}

Of 22 patients receiving prasugrel or ticagrelor, the mean of their post interventional length of stay was 3 days $(S D=6.20)$. (Figure.1) Of 374 patients receiving clopidogrel the mean of the post procedural stay was 1.81 days $(S D=2.67)$. In a multivariate analysis clopidogrel did not affect the patients' post operational length of stay, $p=0.94$. 


\section{DISCUSSION}

Using a retrospective cohort of candidates with cerebrovascular aneurysm(s), we did not identify any association between clopidogrel administration with mortality, thromboembolic accidents, long term recurrence, intra-pipeline stenosis, hemorrhagic events, mRS on latest follow-up and post operational hospital length of stay. We found that clopidogrel is associated with postprocedural complications. Prasugrel and ticagrelor are increasingly adopted in clopidogrel resistant individuals treated for their cerebral aneurysm(s). Compared to clopidogrel, both prasugrel and ticagrelor inhibit platelets aggregation more rapidly and consistently with lower rates of inter-subjects variability. ${ }^{16}$

In the present study, the efficacy of prasugrel, depicted by intra-pipeline stenosis and long-term recurrence, was roughly similar to clopidogrel $(6.1 \%$ vs. $5 \%$ and $1.6 \%$ vs. no recurrence respectively). These results are consistent with the more favorable pharmacokinetic and pharmacodynamic profiles of prasugrel, which affords a more potent and rapid inhibition of platelet aggregation. ${ }^{1}$ They are also in line with the Trial to Assess Improvement in Therapeutic Outcomes by Optimizing Platelet Inhibition with Prasugrel-Thrombolysis In Myocardial Infarction 38 (TRITON-TIMI 38) where clopidogrel-naive patients with acute coronary syndrome scheduled for percutaneous coronary intervention on prasugrel therapy showed significantly reduced rates of ischemic events, including patients with cardiac stent thrombosis. ${ }^{17} \mathrm{~A}$ recent meta-analysis done by Patti et al found that switching from clopidogrel to prasugrel, in patients undergoing percutaneous coronary intervention, tended to decrease the incidence of major adverse cardiac events during follow-up. ${ }^{9}$ Despite the lack of clear evidence supporting its use in cerebrovascular procedures, LeslieMazwi et al were the first to report the successful use of prasugrel for acute in-stent thrombosis in a patient with reduced clopidogrel response undergoing elective stent-assisted aneurysm coiling. ${ }^{7}$

In our series, patients on aspirin and prasugrel did not have any hemorrhagic complications. The small number of patients who were on both aspirin and prasugrel could explain these results, as the use of DAPT with aspirin and prasugrel would be expected to increase the relative risk of bleeding by $30 \%$ compared to aspirin and clopidogrel. 8,17 Interestingly, the greatest benefit with prasugrel vs. clopidogrel in the TRITON-TIMI 38 study was seen in high-risk patients especially diabetics or those who suffered an ST-segment elevation myocardial infarction, where the major adverse cardiac events' reduction with prasugrel was not paralleled by an increased risk of bleeding. ${ }^{9}$ This may infer that there are certain subgroups of patients who are at a decreased risk of the hemorrhagic adverse events from prasugrel use. The incidence of thromboembolic complications was approximately akin in the aspirin/clopidogrel group (7.4\%) and in the aspirin/ prasugrel group (5\%). This was not similar to the extrapolated results of many studies present in the cardiac literature that demonstrated superior reduction of ischemic events using prasugrel as part of DAPT compared to clopidogrel. ${ }^{10,17}$

Ticagrelor/aspirin combination was used only on two patients who either did not achieve the desired P2Y12 platelet inhibition with prasugrel or were allergic to it. One patient had an intra-pipeline stenosis and another suffered from a post-procedural hemorrhagic complication manifesting as mild aphasia. Conclusions about efficacy and safety of ticagrelor in patients with PED cannot be drawn from our series because of our limited number of patients. In their series of 18 patients, Hanel et al presented their successful experience with patients using ticagrelor for different neuroendovascular procedures as an alternative to clopidogrel in nonresponders. ${ }^{5}$ Further investigations in patients undergoing treatment with PED and other neuroendovascular procedures are needed to assess the efficacy and safety profile of ticagrelor in hypo-responders and nonresponders to clopidogrel.

It is noteworthy to state that our series followed during a mean of 15.8 months $(S D=12.4$ months) have showed no regression but an increasingly improvement of the patients' clinical wellbeing. All our patients had a mRS score $<=2$ and their mean length of stay in the hospital was approximately 3 days with
$65 \%$ discharged within two days. This goes in line with the series of Stetler who also was able to discharge his patients on prasugrel on day 1 postoperatively. ${ }^{14}$ We may reckon that prasugrel would not only be efficacious, it could be safe whilst dispensed in this context. This patient-safety model is definitely multifactorial and it might not be plainly related to the use of prasugrel. Although, we might imply that prasugrel would not be adversely interfering with the patient's clinical wellbeing.

There is still no clear indication for the use of prasugrel as an alternative treatment for patients' resistance to clopidogrel during the placement of PED. The main concern of clinicians is the increased bleeding risks associated with its use as shown in several cardiovascular studies. ${ }^{9,17}$ However, the difference in end organ result response (brain vs. cardiac muscle), tortuosity of intracranial vasculature, and amount of metal implanted make it ineffective to simply apply cardiac literature to intracranial procedures. ${ }^{5}$ Akbari et al ${ }^{1}$ presented their experience with prasugrel and aspirin in a cohort of 25 patients undergoing different neuro-endovascular procedures, nine of which undergoing PED placement. They observed a significant increase in hemorrhagic complications $(19.4 \%$ vs. $3.6 \% ; p=0.02)$ in the prasugrel/ aspirin group as compared to patients treated with clopidogrel/aspirin. Jones et al tried using low dose prasugrel in two cases following PED implantation in patients who showed hypo-responsiveness to clopidogrel. Both patients did well with no thromboembolic or hemorrhagic complications. ${ }^{6}$ Our series of patients treated with PED placement who were started on prasugrel due to hypo-responsiveness to clopidogrel is the largest so far. We did not observe any ischemic events related to thromboembolism or in-stent thrombosis. We also did not find an increased risk of bleeding in those patients. Whether our patients fall into a subgroup of patients, which has a lower propensity to have bleeding complications with prasugrel, or these results are due to serendipity alone is not clear. Conclusions cannot be drawn at this level, and more investigations should be warranted to study the efficacy and safety of prasugrel in patients treated endovascularly with PED placement who 
are hypo-responders to clopidogrel. The higher cost of prasugrel compared to clopidogrel should be also taken into consideration when prescribing the drug. ${ }^{4}$

\section{LIMITATIONS}

While our series is one of the largest to date documenting the safety and efficacy of prasugrel in the endovascular pipeline setting, our study design is limited by the small sample size and by the retrospective nature of data collection. None of the patients receiving prasugrel manifested major adverse events. This does not definitively show that prasugrel is as effective as clopidogrel in the pipeline patient population and our results could not be extrapolated to all the neurointervention specialized centers. Further randomized clinical trials are indispensable to display the promising outcome of these drugs in what they could replace clopidogrel in patients receiving PED flow diversion treatment.

\section{CONCLUSION}

The key in assuring clopidogrel resistant patients long term clinical wellbeing is by applying the right anti-aggregation protocol. Approximately $30 \%$ of the patients receiving clopidogrel are heterozygote for the CYP2C19 gene and showing a hypo-responsiveness or resistance $(<30 \%$ platelet inhibition). Prasugrel is to be considered in clopidogrel resistant and allergic patients undergoing flow diversion treatment for their intracranial aneurysms.

\section{REFERENCE}

1. Akbari SH, Reynolds MR, Kadkhodayan Y, Cross DT, 3rd, Moran CJ: Hemorrhagic complications after prasugrel (Effient) therapy for vascular neurointerventional procedures. J Neurointerv Surg 5:337-343, 2013

2. Delgado Almandoz JE, Kadkhodayan $Y$, Crandall BM, Scholz JM, Fease JL, Tubman DE: Variability in initial response to standard clopidogrel therapy, delayed conversion to clopidogrel hyper-response, and associated thromboembolic and hemorrhagic complications in patients undergoing endovascular treatment of unruptured cerebral aneurysms. J Neurointerv Surg 6:767-773, 2014

3. Guédon A, Clarençon F, Maria FD, Rosso C, Biondi A, Gabrieli J, et al: Very late ischemic complications in flow-diverter stents: a retrospective analysis of a single-center series. Journal of Neurosurgery 125:929-935, 2016
4. Gupta R, Moore JM, Griessenauer CJ, Adeeb N, Patel AS, Youn R, et al: Assessment of Dual Antiplatelet Regimen for Pipeline Embolization Device Placement: A Survey of Major Academic Neurovascular Centers in the United States. World Neurosurg, 2016

5. Hanel RA: Safety and efficacy of ticagrelor for neuroendovascular procedures. A single center initial experience. Journal of Neurointerventional Surgery, 2014

6. Jones GM, Twilla JD, Hoit DA, Arthur AS: Prevention of stent thrombosis with reduced dose of prasugrel in two patients undergoing treatment of cerebral aneurysms with pipeline embolisation devices. Journal of Neurolnterventional Surgery 5:e38, 2013

7. Leslie-Mazwi TM, Chandra RV, Oh DC, Nogueira RG: Novel use of prasugrel for intracranial stent thrombosis. Journal of Neurolnterventional Surgery 3:358-360, 2011

8. Mega JL, Close SL, Wiviott SD, Shen L, Hockett RD, Brandt JT, et al: Cytochrome P450 genetic polymorphisms and the response to prasugrel: relationship to pharmacokinetic, pharmacodynamic, and clinical outcomes. Circulation 119:2553-2560, 2009

9. Patti G, Ricottini E, De Luca L, Cavallari I: Safety and Efficacy of Switching From Clopidogrel to Prasugrel in Patients Undergoing Percutaneous Coronary Intervention: A Study-level Meta-analysis From 15 Studies. J Cardiovasc Pharmacol 67:336-343, 2016

10. Paul PD, Sara V, Meagan D: Antiplatelet Agents in Cardiology: A Report on Aspirin, Clopidogrel, Prasugrel, and Ticagrelor. Current Pharmaceutical Design 22:19181932, 2016

11. Pistocchi S, Blanc R, Bartolini B, Piotin M: Flow diverters at and beyond the level of the circle of willis for the treatment of intracrania aneurysms. Stroke 43:1032-1038, 2012

12. Qureshi Al, Luft AR, Sharma M, Guterman LR, Hopkins LN: Prevention and treatment of thromboembolic and ischemic complications associated with endovascular procedures: Part I - Pathophysiological and pharmacological features. Neurosurgery 46:1344-1359, 2000

13. Qureshi Al, Luft AR, Sharma M, Guterman LR, Hopkins LN: Prevention and treatment of thromboembolic and ischemic complications associated with endovascular procedures: Part II--Clinical aspects and recommendations. Neurosurgery 46:1360-1375; discussion 1375-1366, 2000

14. Stetler WR, Chaudhary N, Thompson BG, Gemmete JJ, Maher CO, Pandey AS: Prasugrel is effective and safe for neurointerventional procedures. J Neurointerv Surg 5:332-336, 2013

15. Tan LA, Keigher KM, Munich SA, Moftakhar R, Lopes DK: Thromboembolic complications with Pipeline Embolization Device placement impact of procedure time, number of stents and pre-procedure $\mathrm{P} 2 \mathrm{Y} 12$ reaction unit (PRU) value. J Neurointerv Surg 7:217-221, 2015
16. Weerakkody GJ, Jakubowski JA, Brandt JT, Farid NA, Payne CD, Zhu J, et al: Comparison of speed of onset of platelet inhibition after loading doses of clopidogrel versus prasugrel in healthy volunteers and correlation with responder status. Am J Cardiol 100:331-336, 2007

17. Wiviott SD, Braunwald $\mathrm{E}, \mathrm{McC}$ abe $\mathrm{CH}$, Montalescot G, Ruzyllo W, Gottlieb S, et al: Prasugrel versus Clopidogrel in Patients with Acute Coronary Syndromes. New England Journal of Medicine 357:2001-2015, 2007

\section{Corresponding Author}

Pascal Jabbour, MD

Professor and Director

Division of Neurovascular Surgery and Endovascular Neurosurgery

Department of Neurological Surgery

Thomas Jefferson University Hospital

901 Walnut street 3rd Floor

Philadelphia PA 19107

P: 215-955-7000

F: 215-503-7038

E: pascal.jabbour@jefferson.edu

\section{Co-Authors E-mails and Affiliations}

\section{Elias Atallah}

Thomas Jefferson University,

E: elias.atallah@jefferson.edu

\section{Hassan Saad}

Arkansas Neuroscience Institute,

E: hasan.sa3ed@hotmail.com

\section{Kimon Bekelis}

Thomas Jefferson University

E: kbekelis@gmail.com

\section{Nohra Chalouhi}

Thomas Jefferson University

E: nohra.chalouhi@jefferson.edu

\section{Stavropoula Tjoumakaris}

Thomas Jefferson University

E: stavropoula.tjoumakaris@jefferson.edu

\section{Robert H. Rosennwasser}

Thomas Jefferson University

E: robert.rosenwasser@jefferson.edu

\section{David Hasan}

University of lowa

E: david-hasan@uiowa.edu

\section{Jorge Eller}

Thomas Jefferson University

E: ellerjlahotmail.com

\section{David Stidd}

Thomas Jefferson University

E: david.stidd@jefferson.edu 